\title{
Parallel and vertical morphologies in block copolymers of cylindrical domain
}

\author{
K. Y. Suh, Y. S. Kim, and Hong H. Lee ${ }^{\text {a) }}$ \\ Department of Chemical Engineering and Nanoelectronics Institute, Seoul National University, \\ Seoul, 151-742, Korea
}

(Received 30 June 1997; accepted 9 October 1997)

\begin{abstract}
We present a theoretical result on the ordering of cylindrical morphology in diblock and triblock copolymers. The equilibrium morphology of $A-B$ diblock and $A-B-A$ triblock copolymer films is shown to have the cylinders oriented parallel or vertical to the surface, depending on the film thickness and surface tensions. A criterion is derived that can be used to determine the conditions under which the orientation is parallel or vertical. (C) 1998 American Institute of Physics. [S0021-9606(98)51103-8]
\end{abstract}

\section{INTRODUCTION}

Block copolymers are known to segregate into ordered morphological structures in bulk state. ${ }^{1}$ These regular structures can be spherical, cylindrical, or lamellar depending on the weight fraction of one of the blocks. This process of self-assembly is driven by total free energy minimization, with enthalpic and entropic contributions. Much work has been devoted to the equilibrium bulk phases of block copolymers. $^{2,3}$ In block copolymer thin films, however, there are additional contributions to the overall free energy from the surface and interfacial energies of blocks and substrate. Diblock and triblock copolymers have also been observed to assemble into two- and three-dimensional structures in thin films. $^{4-8}$

Theoretical studies of block copolymer thin films have centered on confined systems. ${ }^{9-11}$ Confined systems do not lead to an island or hole formation. Confinement implies that hills and valleys cannot be formed, making the confined system more amenable to theoretical approaches. Turner ${ }^{10} \mathrm{de}-$ veloped a phenomenological free energy model for the strong segregation limit which considers symmetric and antisymmetric lamellar morphologies organized parallel to the confining surfaces. A recent theoretical paper ${ }^{11}$ revealed that the equilibrium structure of a very thin confined symmetric diblock copolymer may be one in which the lamellae are oriented perpendicular to the surface. However, less theoretical work has been reported on block copolymers of cylindrical domain, perhaps because theoretical complications arise from the three-dimensional nature of the potential which can only be solved numerically.

In thick films, the incompatibility of the film thickness with the cylindrical repeat distance can be distributed over many layers. When the film thickness is of the order of (but not exactly equal to) just a few repeat distances, the structure will become overly distorted with respect to the bulk equilibrium structure. A parallel orientation would require too much elastic energy of polymer chains. Therefore, the equilibrium mesophase structure of very thin films is one in which the cylinders are oriented perpendicular to the surface,

${ }^{a)}$ Author to whom correspondence should be addressed. so that the equilibrium repeat distance can be maintained.

In this paper, we derive an inequality from the bulk equilibrium free energy that can explain the ordering phenomena in block copolymers of cylindrical domain under confinement. We use a simple scalinglike expression for the free energy that is valid in the strong segregation limit. A comparison between the full self-consistent field treatment and the strong segregation limit in thin films can be found in the work of Matsen. ${ }^{12}$

\section{THEORETICAL MODEL}

\section{A. Diblock copolymers of cylindrical domain}

We consider cylindrical diblock copolymers first. The free energy per chain of cylindrical diblock copolymer can be written as follows:

$$
F=F_{1}+F_{c 1}+F_{c 2},
$$

where $F_{i}$ is the interfacial energy per chain at the $A$ block$B$ block interface and $F_{c 1}$ and $F_{c 2}$ are the conformational energies per chain for the inner and the outer block, respectively. They are approximated by the following expressions: ${ }^{13-16}$

$$
F_{c j}=\frac{3}{2} k_{B} T\left[\left(\frac{R_{j}}{R_{j 0}}\right)^{2}+\left(\frac{R_{j 0}}{R_{j}}\right)^{2}-2\right],
$$

where $j$ is the index for the inner or the outer block and $R_{j}$ is the end-to-end distance of block $j$ in the microphaseseparated morphology. In the Alexander-de Gennes ${ }^{15,16}$ formalism this end-to-end distance is taken as the thickness of the layer in the structure corresponding to block $j$. The ideal Gaussian chain end-to-end distance of the $j$ block is denoted by $R_{j 0}$, which is the reference state for the chain conformational energy given by $a_{j} N_{j}^{12}$, where $N_{j}$ is the degree of polymerization of block $j$ and $a_{j}$ is the statistical segment length of the polymer in block $j$. The Wigner-Seitz unit cell for a two-dimensional cylindrical lattice is a hexagon. With $d$ defined as the distance between the centers of the two cylinders (see Fig. 2), $R_{1}$ and $R_{2}$ can be parametrized with a single variable, $d$, as follows: ${ }^{17}$

$$
R_{1}=\left(3^{1 / 2} / 2 \pi\right)^{1 / 2} \phi^{1 / 2} d,
$$




$$
R_{2}=\left(3^{1 / 2} / 2 \pi\right)^{1 / 2}\left(1-\phi^{1 / 2}\right) d,
$$

where $\phi$ is the volume fraction of polymer $A$. The above formalism allows one to forego the usual complicated numerical work resulting from the hexagonal boundary condition. As a result, Eq. (1) for the free energy per chain becomes

$$
\begin{aligned}
F= & F_{i}+F_{c 1}+F_{c 2} \\
= & \gamma_{i} 2 \pi R_{1} \frac{\phi}{\pi R_{1}^{2}} \frac{V}{p}+\frac{3}{2} k_{B} T\left[\left(\frac{R_{1}}{R_{10}}\right)^{2}\right. \\
& \left.+\left(\frac{R_{10}}{R_{1}}\right)^{2}+\left(\frac{R_{2}}{R_{20}}\right)^{2}+\left(\frac{R_{20}}{R_{2}}\right)^{2}-4\right] \\
= & 3.81 \frac{N}{\rho d} \phi^{1 / 2} \gamma_{i}+\frac{3}{2} k_{B} T\left[A d^{2}+\frac{B}{d^{2}}-4\right],
\end{aligned}
$$

where $\rho=p N / V, V$ is the domain volume, $N$ is the degree of polymerization, $p$ is the number of copolymer chains in the system, and $A$ and $B$ are functions of $R_{10}, R_{20}$, and volume fraction, given by

$$
\begin{aligned}
& A=\frac{\sqrt{3} / 2 \pi}{R_{10}^{2}} \phi+\frac{\sqrt{3} / 2 \pi}{R_{20}^{2}}\left(1-\phi^{1 / 2}\right)^{2}, \\
& B=\frac{R_{10}^{2}}{\sqrt{3} / 2 \pi} \frac{1}{\phi}+\frac{R_{20}^{2}}{\sqrt{3} / 2 \pi} \frac{1}{\left(1-\phi^{1 / 2}\right)^{2}} .
\end{aligned}
$$

Equation (5) indicates that the equilibrium lattice constant $\left(d_{0}\right)$ is larger than the ideal Gaussian end-to-end distance. This fact is supported by the experimental results which show that the cylindrical core blocks are stretched by about $10 \%-40 \%$ of their unperturbed end-to-end distance. ${ }^{17,18}$ The amount of stretching in each case depends upon composition, molecular weight, and sample preparation techniques. Although uncertainty remains over the corona block, particularly in the case of strong corona overlap, the outer block chains are also stretched. ${ }^{19}$

For thin films, additional surface effects should be considered. The free energy expressions become

$$
\begin{aligned}
F_{v}= & F_{i}+F_{c 1}+F_{c 2}+F_{s} \\
= & 3.81 \frac{N}{\rho d_{0}} \phi^{1 / 2} \gamma_{i}+\frac{3}{2} k_{B} T\left[A d_{0}^{2}+\frac{B}{d_{0}^{2}}-4\right] \\
& +2 \Sigma\left[\phi \gamma_{1 s}+(1-\phi) \gamma_{2 s}\right]
\end{aligned}
$$

for vertical morphology and

$$
\begin{aligned}
F_{p} & =F_{i}+F_{c 1}+F_{c 2}+F_{s} \\
& =3.81 \frac{N}{\rho d} \phi^{1 / 2} \gamma_{i}+\frac{3}{2} k_{B} T\left[A d^{2}+\frac{B}{d^{2}}-4\right]+2 \Sigma \gamma_{1 s}
\end{aligned}
$$

for parallel morphology. Note in this regard that the equilibrium period can be realized in the vertical morphology whereas it cannot be in the parallel morphology. Here, $F_{s}$ is the surface energy, $\Sigma$ is the contact area per chain at the polymer-substrate interface, and $\gamma_{j s}$ is the interfacial tension

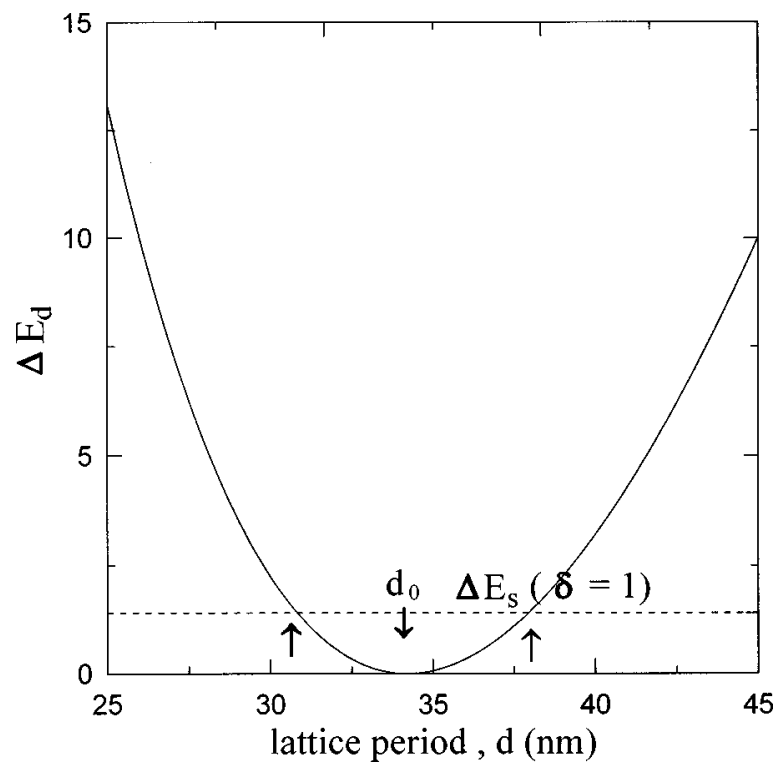

FIG. 1. Relationship between the lattice period, $d$, and $\Delta E_{s}$. Note that $\Delta E_{s}$ has a minimum at $d=d_{0}$. The arrows indicate the transition from parallel to vertical morphology.

between chain $j$ and the substrate. In the parallel morphology, the copolymer chain of lower surface tension covers the substrate, particularly when one of the blocks has a stronger interaction with the substrate than the other. A surfaceinduced transition from cylindrical to lamellar morphology was predicted by several authors. ${ }^{20-22}$ Turner et al. ${ }^{22}$ for instance, found that a region of surface-induced lamellar ordering could occur when the difference in the surface tensions of the $A$ and $B$ polymers with respect to the substrate is larger than a critical value. They also note that this condition is readily accessible experimentally. ${ }^{7}$

To obtain a criterion for vertical morphology, it is sufficient to set $F_{p}-F_{v}>0$. Thus, we have from Eqs. (8) and (9)

$$
\begin{aligned}
\frac{F_{p}-F_{v}}{\gamma_{i} \Sigma}= & \Delta E_{d}-\Delta E_{s} \\
= & 3.81 \omega \phi^{1 / 2}\left(\frac{d_{0}}{d}-1\right)+\frac{3}{2} \epsilon\left[A d_{0}^{2}\left(\left(\frac{d}{d_{0}}\right)^{2}-1\right)\right. \\
& \left.+B \frac{1}{d_{0}^{2}}\left(\left(\frac{d_{0}}{d}\right)^{2}-1\right)\right]-2(1-\phi) \delta>0
\end{aligned}
$$

where the dimensionless parameters are

$$
\omega=\frac{N}{\Sigma \rho d_{0}}, \quad \epsilon=\frac{k_{B} T}{\gamma_{i} \Sigma}, \quad \delta=\frac{\gamma_{2 s}-\gamma_{1 s}}{\gamma_{i}} .
$$

In Eq. (10), the first two terms represent $\Delta E_{d}$, which is the energy difference arising from the lattice period of block copolymer of parallel orientation being different from the equilibrium one for thin films. On the other hand, $\Delta E_{s}$, represented by the last term in Eq. (10), is the energy difference resulting from the surface effect.

Figure 1 shows $\Delta E_{d}$ that is dependent on the lattice period, which reaches the minimum at $d=d_{0}$. Typical parameter values used in Fig. 1 are: $\omega=1, \epsilon=10, R_{10}=8 \mathrm{~nm}$, 


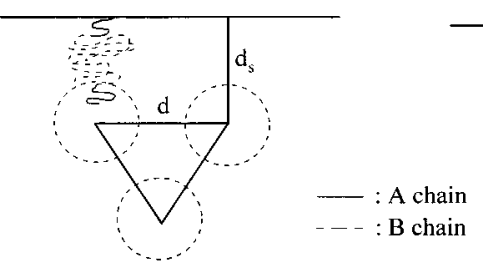

(a) (b)

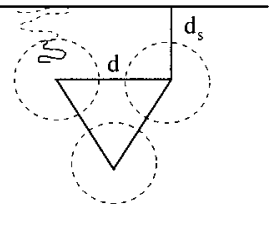

FIG. 2. Schematic diagram of surface morphology of diblock copolymers where $d_{s}$ and $d$ are defined. (a) $\gamma_{1 s}<\gamma_{2 s}$ (b) $\gamma_{1 s}>\gamma_{2 s}$.

$R_{20}=10 \mathrm{~nm}, \phi=0.3$. The minimum, which corresponds to the equilibrium period, $d_{0}$, is $34.3 \mathrm{~nm}$. Thus, the inner core block is stretched about $23 \%$ from its equilibrium value. As is indicated in Fig. 1, the higher the surface effect is, the more difficult it is for the vertical morphology to form.

The lattice period of parallel morphology is related to the film thickness. Although it is difficult to quantitatively relate the thickness of the surface lamellar layer to the material property, volume fraction, and surface tensions, the film thickness can nevertheless be expressed as follows:

$$
\begin{array}{rlrl}
t & =2 d_{s}+n d \sin 60^{\circ} & t & =2 d_{s}+(n+1) d \sin 60^{\circ} \\
& =2 d_{s}+(n+\alpha) d_{0} \sin 60^{\circ} & =2 d_{s}+(n+\alpha) d_{0} \sin 60^{\circ},
\end{array}
$$

where $d_{s}$ is defined in Fig. 2. Then the lattice period of a thin film is related to the equilibrium period by

$$
(n+\alpha) d_{0}=n d \quad \text { or }(n+\alpha) d_{0}=(n+1) d,
$$

where $n$ or $n+1$ layer is possible depending on the given film thickness. The use of Eq. (13) in Eq. (10) leads to the following more tractable criterion:

$$
\begin{aligned}
& -3.81 \omega \phi^{1 / 2} \frac{\alpha}{n+\alpha}+\frac{3}{2} \epsilon\left[A d_{0}^{2} \frac{\alpha(2 n+\alpha)}{n^{2}}\right. \\
& -B \frac{1}{d_{0}^{2}} \frac{\alpha(2 n+\alpha)}{(n+\alpha)^{2}}>2(1-\phi) \delta \text { for } n \text { layer, } \\
& 3.81 \omega \phi^{1 / 2} \frac{1-\alpha}{n+\alpha}+\frac{3}{2} \epsilon\left[-A d_{0}^{2} \frac{(1-\alpha)(2 n+1+\alpha)}{(n+1)^{2}}\right. \\
& +B \frac{1}{d_{0}^{2}} \frac{(1-\alpha)(2 n+1+\alpha)}{(n+\alpha)^{2}}>2(1-\phi) \delta
\end{aligned}
$$

for $n+1$ layer.

The thickness range (cross-hatched portion) in which the vertical morphology results is shown in Fig. 3 for the parameters given there. The quantity $\alpha$ is a fractional number that results when the film thickness is related to the equilibrium lattice constant. For a given film thickness, there can be $n$ or $(n+1)$ number of repeating layers. At certain thicknesses, the energy minimization drives the system to one in which $(n+1)$ number of layers is more energetically stable than the $n$ number of layers. Hence, an abrupt transition occurs from $n$ number of layers to $(n+1)$ number of layers as shown in Fig. 3. As a result, the thickness range in which the vertical

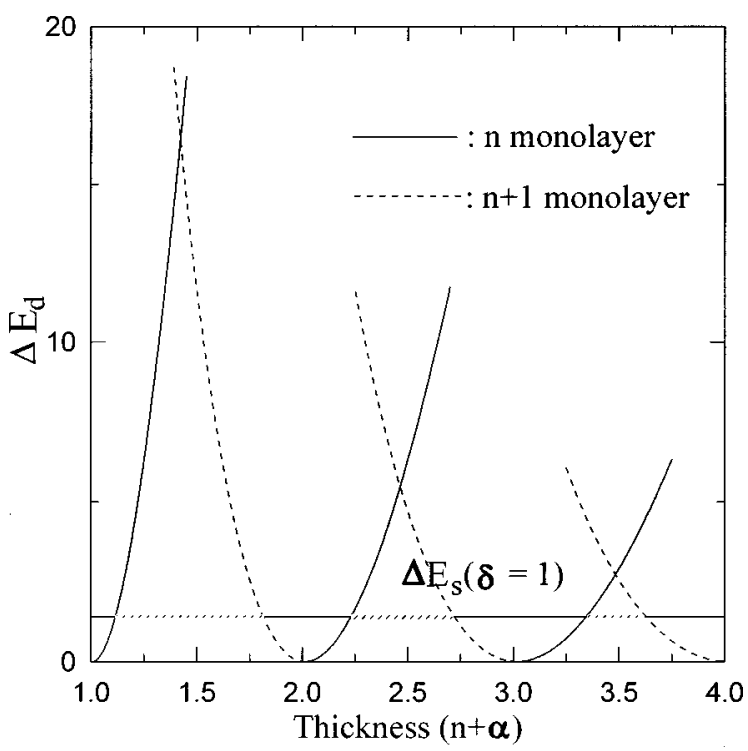

FIG. 3. Thickness range (cross hatched) for vertical morphology. The parameter values are: $\omega=1, \epsilon=10, R_{10}=8 \mathrm{~nm}, R_{20}=10 \mathrm{~nm}, \phi=0.3, d_{0}$ $=34.3 \mathrm{~nm}$, and $\delta=1$. Note that the range for vertical morphology increases as the thickness decreases.

morphology exists is not continuous but rather disjointed (see the cross-hatched portion), jumping from one range to another as the thickness increases. This range decreases with increasing thickness. The midtransition point gradually approaches the midpoint between $n$ and $n+1$ as the thickness increases. As shown in the diagram, the vertical morphology does not form for thick films and the window for morphology decreases with decreasing surface effect, or $\Delta E_{s}$, as expected.

\section{B. Triblock copolymers of cylindrical domain}

In order to extend to triblock copolymers, additional consideration must be given to bulk and surface effects. A schematic diagram of a triblock surface configuration is shown in Fig. 4. In the case of the $A B A$ triblock copolymer in which the $A$ chain (inner core block) has a lower surface tension than the $B$ chain, we assume that the surface constraint is nearly the same as that of the diblock copolymer. However, when the $B$ chain has a lower surface tension than the $A$ chain, a certain fraction of the $A$ chain exists in the surface, resulting in a modified surface energy:

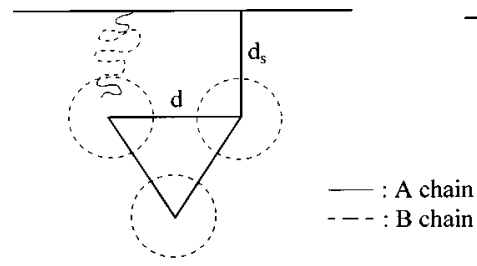

(a)

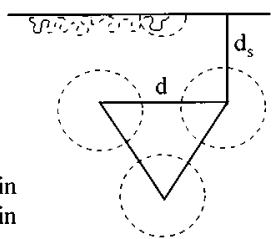

(b)
FIG. 4. Schematic diagram of surface morphology of triblock copolymers: (a) $\gamma_{1 s}<\gamma_{2 s}$ (b) $\gamma_{1 s}>\gamma_{2 s}$. In the case of (b), some fraction of the $A$ chain is in contact with the substrate. 


$$
F_{s}=2 \Sigma\left[\phi_{s} \gamma_{1 s}+\left(1-\phi_{s}\right) \gamma_{2 s}\right] \text {, }
$$

where $\phi_{s}$ is the surface fraction of $A$ chain. For the parallel morphology to be energetically stable, $\phi_{s}$ should be smaller than the bulk fraction $\phi$. But a much smaller value is not favorable because of a large deformation of chain that can result at the surface. Consequently, there is a window of $\phi_{s}$ for the energetically stable parallel morphology.

It should be noted here that if the midblock has a very strong interaction with a substrate such as a hydrogen bonding, the end block is driven away from the surface through a looping process and there is no end chain at the surface. Experimental result supports this phenomenon. It is known, for instance, that one of the blocks that forms the hydrogen bonding with the substrate occupies the surface. ${ }^{7}$ Therefore, our results are restricted to the system where the difference between the two surface tensions is not large enough to overcome the additional energy required of the looping process, as in the styrene-butadiene-styrene (SBS) triblock copolymer. There could be a loop fraction in the bulk state but this energy is canceled out in the process of taking the energy difference between the two morphologies.

For $A B A$ triblock copolymers in which the $B$ chain has a smaller surface tension than the $A$ chain, the criterion can easily be modified and compared with the diblock copolymers. In general, the inequality condition lowers $\Delta E_{s}$, which results in an increase of the thickness range for vertical morphology.

\section{COMPARISON WITH EXPERIMENTAL RESULTS AND DISCUSSION}

Comparison of the results with the experimental data of diblock copolymers in the literature is limited in that the data are not available. It has been reported that only parallel morphology can occur. ${ }^{4,6}$ In order to produce the thin films with cylinders oriented perpendicular to the substrate, Mansky et $a l .{ }^{4}$ spread a drop of diluted polymer solution in toluene on the surface of a de-ionized water bath, allowing the solvent to evaporate. But the process is not an equilibrium one and is therefore metastable. Our results show that if the difference between two surface tensions is not large and the thickness can be controlled well below the several repeat distances, vertical morphology will result.

In triblock copolymers, Dijk et $a l .{ }^{8}$ reported that a relatively thick $(140 \mathrm{~nm})$ film of SBS triblock copolymer has the
PS cylinders oriented parallel to the substrate surface whereas a thin $(42 \mathrm{~nm})$ film has them oriented vertical to the surface. Our theory can explain the data satisfactorily in that the material is $A B A$ type in which the midblock has a lower surface tension than the end block. In this case, $\Delta E_{s}$ has a much lower value than in the case of the diblock, which facilitates the vertical morphology. On the other hand, Liu et $a l^{7}$ reported that in the cylindrical deuterated polystyrene(dPS)-poly(vinylpyridine)(PVP) system, only parallel morphology was observed regardless of the thickness. In this system, poly(vinylpyridine) has so strong an interaction with the silicon oxide substrate that the morphology is dominated by the surface effects, making only the parallel morphology possible.

In summary, a criterion has been established for determining whether the orientation for the block copolymers of cylindrical domain is parallel or vertical to the substrate surface. The introduction of a single parameter, $d$, has been proven to greatly simplify the prediction of the orientation dependence of thin film.

${ }^{1}$ F. S. Bates and G. H. Fredrickson, Annu. Rev. Phys. Chem. 41, 525 (1990).

${ }^{2}$ E. Helfand, Macromolecules 8, 552 (1975).

${ }^{3}$ T. Ohta and K. Kawasaki, Macromolecules 19, 2621 (1986).

${ }^{4}$ P. Mansky, P. Chaikin, and E. L. Thomas, J. Mater. Sci. 30, 1987 (1995).

${ }^{5}$ S. H. Anastasiadis, T. P. Russell, S. K. Satija, and C. F. Majkrzak, Phys. Rev. Lett. 62, 1852 (1989).

${ }^{6}$ A. Karim, N. Singh, M. Sikka, F. S. Bates, W. D. Dozier, and G. P. Felcher, J. Chem. Phys. 100, 1620 (1994).

${ }^{7}$ Y. Liu et al., Macromolecules 27, 4000 (1994).

${ }^{8}$ M. A. van Dijk and R. van den Berg, Macromolecules 28, 6773 (1995).

${ }^{9}$ K. R. Shull, Macromolecules 25, 2122 (1992).

${ }^{10}$ M. S. Turner, Phys. Rev. Lett. 69, 1788 (1992).

${ }^{11}$ D. G. Walton, G. J. Kellogg, A. M. Mayes, P. Lambooy, and T. P. Russell, Macromolecules 27, 6225 (1994).

${ }^{12}$ M. W. Matsen, J. Chem. Phys. 106, 7781 (1997).

${ }^{13}$ L. Leibler, H. Orland, and J. C. Wheeler, J. Chem. Phys. 79, 3550 (1983).

${ }^{14}$ S. P. Gido, D. W. Schwark, and E. L. Thomas, Macromolecules 26, 2636 (1993).

${ }^{15}$ P. G. de Gennes, J. Phys. (Paris) 37, 1443 (1976).

${ }^{16}$ S. J. Alexander, J. Phys. 38, 977 (1977).

${ }^{17}$ E. Helfand and Z. R. Wasserman, Macromolecules 13, 994 (1980).

${ }^{18}$ G. Hadziioannou and A. Skoulis, Macromolecules 15, 267 (1982).

${ }^{19}$ A. N. Semenov, Sov. Phys. JETP 61, 733 (1985).

${ }^{20}$ K. R. Shull, Macromolecules 26, 2346 (1993).

${ }^{21}$ S. H. Anastasiadis, T. P. Russell, S. K. Satija, and C. F. Majkrzak, J. Chem. Phys. 92, 5677 (1990).

${ }^{22}$ M. S. Turner, M. Rubinstein, and C. M. Marques, Macromolecules 27, 4986 (1994) 